\title{
Estado de hidratação, peroxidação lipídica e pressão arterial em atletas amadores de ironman® 70.3
}

\author{
Kenji Fuke ${ }^{1}$, Vanderson Luis Moro², Camila de Campos Velho Gewehr ${ }^{3}$, Maria Fátima Glaner
}

\begin{abstract}
RESUMO
Comparar o estado de hidratação, dano muscular, peroxidação lipídica e pressão arterial em triatletas amadores, antes e após uma prova de Ironman® 70.3. 0 grupo de estudo foi composto por doze (10 homens e duas mulheres) triatletas amadores ( $34 \pm 8,3$ anos). As variáveis foram mensuradas momentos antes e após a prova de Ironman® 70.3. A massa corporal e a pressão arterial reduziram significantemente $(p<0,05)$. A água corporal relativa, a creatina quinase total (CK total) e suas frações músculo esquelético e cardíaco, cálcio e substâncias reativas ao ácido tiabarbitúrico (TBARs) foram significantemente $(p<0,05)$ maiores após a prova. As demais variáveis (água corporal total, sódio e potássio) não alteraram ( $p>0,05)$ após a competição. A reposição hidroeletrolítica ao longo da prova foi suficiente para manter os triatletas hidratados. Entretanto, a prova de Ironman® 70.3 ocasionou o desgaste da musculatura esquelética, formação de radicais livres e hipotensão nos triatletas, alterações esperadas para atletas desta modalidade esportiva.
\end{abstract}

Descritores: Desempenho Atlético; Hidratação; Radicais Livres.

\section{Hydration status, lipid peroxidation and blood pressure in amateur athletes of Ironman ${ }^{\circledR} 70.3$}

\begin{abstract}
The aim of the study was to compare the hydration status, muscle damage, lipid peroxidation and blood pressure in amateur triathletes before and after an Ironman® 70.3. The study group was composed of twelve (10 men and two women) amateur triathletes (34 \pm 8.3 years). The variables were measured before and after the Ironman® 70.3. Body weight and blood pressure decreased significantly ( $p<0.05)$. The relative body water, creatine kinase (CK), creatine kinase muscle-brain fraction (CK-MB), creatine kinase skeletal muscle fraction (CK-MM), calcium and thiobarbituric acid-reactive substances (TBARs) were significantly $(p<0.05)$ higher after the competition. The other variables (body water, sodium and potassium) did not change $(p>0.05)$ after the competition. The hydroeletrolitic replacement during the test was sufficient to maintain the hydrated triathletes. However, the Ironman ${ }^{\circledR} 70.3$ occasioned damage skeletal muscle, lipid peroxidation and hypotension in triathletes, expected changes for athletes of this sport.
\end{abstract}

Descriptors: Athletic Performance; Hydration; Free Radicals.

\footnotetext{
${ }^{1}$ Mestre em Treino de Alto Rendimento pela Universidade Técnica de Lisboa (UTL), Lisboa, Portugal.

${ }^{2}$ Mestre em Educação Física pela Universidade Federal de Santa Catarina (UFSC), Florianópolis, SC, Brasil.

${ }^{3}$ Doutora em Biomedicina pelo Instituto de Ensino e Pesquisa da Santa Casa de Belo Horizonte (IEP-SCBH), Belo Horizonte, MG, Brasil.

${ }^{4}$ Doutora em Ciência do Movimento Humano pela Universidade Federal de Santa Maria (UFSM), Santa Maria, RS, Brasil.
} 


\section{Introdução}

No universo esportivo, uma fatia considerável de atletas participa de provas de longa duração. 0 triatlon está entre elas, o qual é subdividido em três etapas: natação, ciclismo e corrida. As provas são diferenciadas quanto à distância. 0 Ironman® envolve 3,8 km de natação, $180 \mathrm{~km}$ de ciclismo e 42,19 km de corrida. Uma variação desta modalidade que vem ganhando popularidade é o Meio Ironman® $(1,9 \mathrm{~km}$ de natação, $90 \mathrm{~km}$ de ciclismo e $21 \mathrm{~km}$ de corrida), também conhecido como Ironman $₫ 70.3$ - equivalente, em milhas, à metade do Ironman ${ }^{\mathrm{TM}}$.

Uma vez que estas competições se enquadram dentro das modalidades que resultam em elevado estresse físico, devido ao longo tempo de atividade e condições ambientais muitas vezes adversas, como a longa exposição solar e elevadas temperaturas ${ }^{1}$. Assim sendo, vários sintomas adversos são comumente associados a este tipo de prova, dentre eles estão a hiponatremia, as câimbras, lesões musculoesqueléticas, hipotensão, entre outras' .

A hiponatremia é causada pela redução dos níveis de sódio no sangue. Dentre as possíveis causas cita-se o consumo excessivo de água e a falha no sistema renal ${ }^{2,3}$. No entanto, a ausência de reposição hídrica pode levar o desportista a hipernatremia e, consequentemente, ao estresse térmico, causando câimbras, tonturas, desmaios e redução da capacidade de rendimento ${ }^{4}$. A homeostasia hídrica corporal pode ser monitorada durante uma prova pela diferença entre a massa corporal inicial e final, ou por impedância bioelétrica. Diminuições de cálcio e potássio ${ }^{6,7}$ também influenciam o desempenho, pois intervêm no processo de contração e relaxamento das fibras musculares durante o exercício. Além destes, o aumento do dano muscular e a produção de radicais livres, representados no presente estudo pela creatina quinase (CK) e substâncias reativas ao ácido tiobarbitúrico (TBARs), respectivamente, podem contribuir para uma diminuição no desempenho físico e aparecimento de fadiga ${ }^{8-10}$.

ACKapresenta três diferentes isoformas que fornecem informações sobre danos teciduais específicos. ACK-MM é abundante no músculo esquelético e seu aumento no plasma sugere lesão muscular esquelética. A CK-MB tem alta atividade no músculo cardíaco e seu aumento plasmático sugere lesão do miocárdio. A CK-BB é predominante encontrada no cérebro, pulmões, estômago e bexiga, sendo a sua atividade indetectável no plasma, a não ser nos casos de acidente vascular cerebral grave ${ }^{11}$.

Diante deste contexto, analisar estas variáveis durante o triatlo resultará em informações importantes sobre os riscos e benefícios que o desportista está sujeito durante as provas. Por meio das informações obtidas poder-se-á estabelecer estratégias que minimizem os possíveis efeitos deletérios advindos deste esporte. Estas informações serão fundamentais principalmente para o atleta amador, uma vez que este não tem suporte profissional adequado para a prática da modalidade. Nesse sentido, o objetivo foi comparar o estado de hidratação, dano muscular, peroxidação lipídica e pressão arterial em triatletas amadores, antes e após uma prova de Ironman® 70.3.

\section{Metodologia}

\section{$\underline{\text { Sujeitos }}$}

O grupo de estudo foi composto por atletas amadores do Ironman® 70.3, Brasília-DF. Os sujeitos foram convidados a participar do estudo um dia antes da competição, durante o Congresso Técnico. Trinta e um sujeitos se apresentaram como voluntários. No entanto, somente 12 triatletas (idade média de $34 \pm 8,3$ anos), sendo 10 homens e duas mulheres, foram considerados, pois os demais não compareceram para fazer os testes e coleta de sangue imediatamente após a prova. Este estudo é derivado de um projeto aprovado pelo Comitê de Ética e Pesquisa da Universidade Católica de Brasília, estando, na época de coleta de dados, de acordo com a resolução 196/96 do Conselho Nacional de Saúde.

\section{$\underline{\text { Instrumentos e Procedimentos }}$}

No Congresso Técnico da competição foram esclarecidos os objetivos do estudo e como seria feita a coleta de dados. No dia da competição, à medida que os atletas chegavam ao local da prova, foram novamente convidados a participar da coleta de dados pré-competição (1 hora antes da prova) e pós-competição (30 minutos após o término da prova). A competição teve início as 07 horas e término as 16 horas.

Todos os voluntários assinaram um termo de consentimento livre e esclarecido, que lhes garantia o total anonimato e sigilo quanto aos resultados individuais. Também lhes dava o direito de desistir da participação na pesquisa a qualquer instante, sem qualquer recriminação por parte dos pesquisadores. 
Durante todo o percurso da prova, todos os voluntários tiveram livre acesso ao consumo de água, repositores hidroeletrolíticos, bebidas glicosadas e comidas (ex: pão, banana, glicogel). Não foi estabelecido nenhum limite de consumo destes.

A temperatura média no dia da competição, assim como a umidade relativa do ar não foram verificadas, tornandose limitações desse estudo.

\section{Variáveis mensuradas}

As variáveis foram mensuradas nesta ordem: 1) massa corporal, estatura, impedância bioelétrica, pressão arterial e coleta sanguínea. Exceto a estatura, todas as variáveis foram mensuradas antes e após a competição. A estatura e a massa corporal foram mensuradas seguindo um protocolo padronizado ${ }^{13}$. A água corporal total $(\mathrm{L})$ e relativa $(\%)$ foi estimada pela técnica da impedância bioelétrica (Biodynamics 310), sendo seguidas todas as instruções do manual do referido equipamento, para a coleta pré prova. Para a coleta posterior o protocolo não foi seguido, dada a característica do delineamento do estudo. A pressão arterial foi medida no braço esquerdo pelo método auscultatório utilizando um esfigmomanômetro aneróide (marca PREMIUM com dimensões de braçadeira $145 \times 530$ × 10 mm e de manômetro $52 \times 98 \times 35,5 \mathrm{~mm}$ ) e um estetoscópio (marca PREMIUM). Antes da medida cada sujeito ficou deitado por $10 \mathrm{~min}$, sendo a medida realizada nesta posição.

A coleta sanguínea foi feita por uma pessoa habilitada (COREN-GO 152370) e habituada a tal procedimento. Durante a coleta os voluntários permaneceram sentados, com o braço apoiado sobre um suporte. Em seguida, tiveram seu braço garroteado aproximadamente no ponto médio do úmero e, feita a anti-sepsia do local da coleta com algodão embebido em álcool.

Com a ajuda de um adaptador de agulhas para coletas múltiplas, foi introduzida uma agulha descartável de $25 \times 8$ $\mathrm{mm}$ em uma das veias da fossa antecubital do braço. Aproximadamente $2 \mathrm{~mL}$ de sangue foram coletados em um tubo (Bencton Dickinson) a vácuo. Estes tubos foram colocados em uma caixa térmica com gelo, para depois serem levados ao laboratório onde o sangue foi centrifugado (Centrifuga Celm - LS-3 Plus) no máximo $2 \mathrm{~h}$ depois da sua coleta. A centrifugação foi feita durante $15 \mathrm{~min}$ a $3200 \mathrm{rpm}$. Depois de separado o plasma, este foi armazenado, por uma semana, a uma temperatura entre $0^{\circ}$ e $4^{\circ} \mathrm{C}$. Os mesmos procedimentos de coleta sanguínea foram repetidos no máximo 20 min depois de concluída a última prova da competição.

ATBARs foi estimada espectrofotometricamente conforme descrito por Draper e Hadley ${ }^{14}$. ACK pelo método enzimático através de um auto-analisador bioquímico Targa bt $3000^{\circledR}$. Os reagentes bioquímicos adotados foram da marca Wienner $l a b^{\circledast}$. Foram seguidos todos os procedimentos descritos pelos fabricantes do auto-analisador e dos reagentes. Cada amostra de sangue passou duas vezes pelo auto-analisador e, em caso de não haver equivalência de medida (variação de até $5 \mathrm{U} / \mathrm{L}$ ) seria realizada uma terceira análise, o que não foi o caso. Foram usados os dados da primeira análise. 0 sódio, o potássio e o cálcio foram quantificados por fotometria de chama (Benfer ${ }^{\circledR}$ ).

\section{Análise estatística}

O teste de Shapiro-Wilk foi utilizado para testar a normalidade da distribuição dos dados. $O$ teste $t$ de Student para amostras pareadas e 0 teste de Wilcoxon foram utilizados para comparar as variáveis bioquímicas dos triatletas entre 0 início e o final da prova de triatlo. 0 nível de significância adotado foi de $p \leq 0,05$. A magnitude das diferenças entre 0 início e o final da prova de triatlo foi mensurada pelo tamanho do efeito, sendo este classificado em insignificante $(<0,25)$, pequeno $(0,25-0,49)$, moderado $(0,5-1,0)$ e grande $(>1,0)^{15}$. 0 dados foram analisados nos software SPSS versão 15.0 para Windows (SPSS Inc., Chicago IL, EUA) e G Power 3 (Heinrich-Heine-Universität, Dusseldorf, Alemanha).

\section{Resultados}

As variáveis bioquímicas dos triatletas investigados são descritas na Tabela 1, em forma de valores médios, desviopadrão $( \pm)$, variância média percentual, significância, poder do teste e tamanho do efeito. Pode ser observado que após o Ironman® 70.3 houve uma diminuição significativa $(p<0,05)$ da massa corporal $(2,9 \%)$ e aumento $(p<0,05)$ para a água corporal relativa (1\%), cálcio (15,1\%), CK total (306,4\%), CK MM (328,1\%), CK MB $(45,7 \%)$ e TBARs $(18,2 \%)$. As demais variáveis (água corporal total, sódio e potássio) não se mostram alteradas $(p>0,05)$ após a competição. 
Tabela 1 - Comparação das variáveis bioquímicas pré e pós prova de Ironman® 70.3 .

\begin{tabular}{|c|c|c|c|c|c|c|}
\hline \multirow[t]{2}{*}{ Variáveis } & Pré prova & Pós prova & \multirow[t]{2}{*}{$\Delta \%$} & \multirow[t]{2}{*}{$\mathrm{p}$} & \multirow[t]{2}{*}{ Poder do teste } & \multirow{2}{*}{$\begin{array}{c}\text { Tamanho do } \\
\text { efeito }\end{array}$} \\
\hline & Média \pm & Média \pm & & & & \\
\hline Massa corporal (kg) & $69,2 \pm 11,2$ & $67,2 \pm 11,2$ & $-2,9$ & $<0,001$ & 0,99 & Grande \\
\hline Água corporal total (I) & $37,8 \pm 5,2$ & $38,5 \pm 5,5$ & $+1,8$ & 0,202 & 0,33 & Pequeno \\
\hline Água corporal (\%) & $69,4 \pm 1,7$ & $70,1 \pm 1,6$ & $+1,0$ & 0,011 & 0,89 & Moderado \\
\hline Sódio (mmol/l) & $155 \pm 13,9$ & $155,4 \pm 18,4$ & $+0,2$ & 0,958 & 0,06 & Insignificante \\
\hline Potássio (mmol/l) & $37,5 \pm 4,3$ & $36,3 \pm 3,4$ & $-3,2$ & 0,518 & 0,25 & Pequeno \\
\hline Cálcio (mmol/l) & $19,2 \pm 1,7$ & $22,1 \pm 1,2$ & $+15,1$ & $<0,001$ & 0,99 & Grande \\
\hline $\mathrm{CK}_{\text {(U/L) }}$ & $108 \pm 37,8$ & $439,5 \pm 191,5$ & $+306,4$ & $<0,001$ & 0,99 & Grande \\
\hline $\mathrm{CK} \mathrm{MB}_{\text {(U/L) }}$ & $8,1 \pm 2,9$ & $11,8 \pm 4$ & $+45,7$ & 0,011 & 0,89 & Moderado \\
\hline $\mathrm{CK} \mathrm{MM}_{\text {(ULL) }}$ & $99,9 \pm 37$ & $427,7 \pm 189,8$ & $+328,1$ & $<0,001$ & 0,99 & Grande \\
\hline TBARs ( $\mu \mathrm{mol} \mathrm{MDA} / \mathrm{ml})$ & $0,242 \pm 0,06$ & $0,286 \pm 0,04$ & $+18,2$ & 0,03 & 0,74 & Moderado \\
\hline
\end{tabular}

CK: creatina quinase; MB: fração da enzima CK referente à musculatura estriada cardíaca; MM: fração da enzima CK referente à musculatura esquelética; TBARs: Substâncias reativas ao ácido tiobarbitúrico.

Houve diferença $(p<0,05)$ na PAS (Pré: $121,05 \pm 8,75$ mmHg e Pós: $99,66 \pm 13,25 \mathrm{mmHg}$ ) e PAD (Pré: 78,94 $\pm 8,09$ mmHg e Pós: $69,33 \pm 8,68 \mathrm{mmHg}$ ) dos triatletas amadores quando comparados os seus valores do início e final do Ironman® 70.3, conforme ilustrado na Figura 1.

Figura 1 - Valores médios de pressão arterial sistólica (PAS) e diastólica (PAD) de triatletas antes e após a prova de Ironman® 70.3.

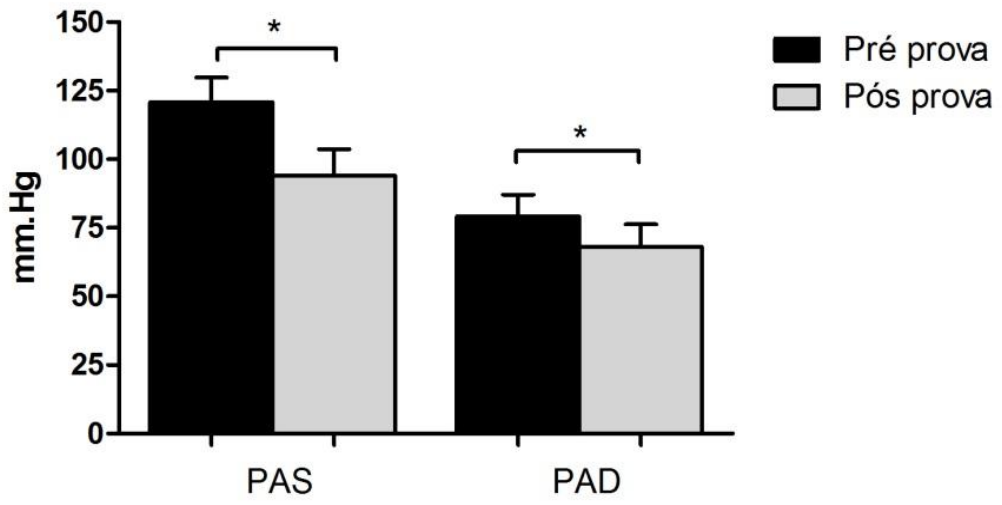

${ }^{*}$ Representa diferença entre pré e pós prova de Ironman® 70.3 (Teste t de Student para amostras pareadas) $p \leq 0,05$.

\section{Discussão}

Em provas como o Ironman® 70.3, participam muitos atletas amadores, que na sua maioria não tem uma orientação adequada para tal, principalmente no que se refere a determinadas variáveis bioquímicas, indicadoras de determinados riscos, como exemplo o dano muscular e a hiponatremia. Assim, analisar tais variáveis em atletas amadores, em uma situação real, antes e após uma prova de Ironman ${ }^{\circledR} 70.3$, pode elucidar a tal grupo populacional os possiveis riscos desta prática sem um monitoramento adequado.

Inicialmente os resultados demonstraram uma perda de 2,9\% na massa corporal. Alguns estudos ${ }^{16-18}$ evidenciaram que tal perda está atrelada a desidratação, outros ${ }^{19-21}$ afirmam que é devida à oxidação da gordura corporal e a diminuição das reservas de glicogênio, à liberação da água armazenada com o glicogênio muscular e hepático. Essas alterações foram observadas por Knechtle et a ${ }^{19}$ que investigaram as mudanças de massa corporal total, massa gorda, massa muscular e a água corporal total de 31 ultra-triatletas amadores. Os resultados mostram que a diminuição da massa corporal total foi associada à diminuição da massa muscular e da massa gorda, enquanto que a água corporal total não mudou $(p>0,05)$ após a prova Triple Iron Triathlon (11,4 km de natação, 540 km de ciclismo e 126,6 km de corrida). 
Inversamente a diminuição da massa corporal foi observado um aumento da água corporal relativa (Tabela 1). Esses achados mostram que o nível de hidratação não foi a causa da diminuição da massa corporal dos triatletas amadores ao final do Ironman ${ }^{\circledR}$ 70.3. Assim, corroborando com outras investigações ${ }^{19-22}$, acredita-se que essa diminuição deriva provavelmente de outras fontes (oxidação da gordura corporal e a diminuição das reservas de glicogênio) que não a perda de líquidos.

A ingestão de grandes quantidades de água não é suficiente para a reposição hidroeletrolítica devido à sudorese excessiva e uma consequente perda de eletrólitos, fato que acontece em provas de Ironman® 70.3. Para tanto, uma ingestão adequada de fluidos contendo, principalmente, sódio e potássio deve fazer parte do processo de reidratação. No presente estudo (Tabela 1) não houve alteração $(p>0,05)$ na concentração de sódio e potássio no sangue.

Apesar da concentração de sódio estar acima dos valores normais de referência (135 - $145 \mathrm{mmol} / \mathrm{L})$, Howanitz e Howanitz ${ }^{23}$ ressaltam que os valores críticos do sódio são $<120 \mathrm{mmol} / \mathrm{L}$ (hiponatremia) e $>155 \mathrm{mmol} / \mathrm{L}$ (hipernatremia). Ainda que os triatletas amadores deste estudo apresentem concentrações de sódio muito próximas aos valores críticos para a ocorrência de hipernatremia, está não foi observada, pois não houve alterações da concentração do sódio no final da prova e nem desidratação. Essa concentração dentro dos limites aceitáveis favorece a transmissão de impulsos nervosos e o funcionamento dos músculos acontece de maneira mais adequada, sendo que uma diminuição na concentração de sódio no sangue pode alterar essas funções.

Em relação às alterações da concentração de sódio sanguíneo em provas de triatlo, os estudos ainda são controversos. BürgerMendonça et a ${ }^{24}$ analisaram os níveis séricos de sódio em seis triatletas amadores antes e após o Ironman® 70.3. Os autores encontraram uma diminuição $(p<0,05)$ no sódio sanguíneo, porém não houve hiponatremia, pois os resultados permaneceram dentro de valores de referência. Em triatletas participantes das provas de Ironman ${ }^{\top \mathrm{M}}$, foi observado um aumento significativo $(p<0,05)$ naqueles que possuíam uma perda de massa corporal acima de $5 \%$, porém nos que apresentaram redução de $3 \%$ de massa corporal, similar ao presente estudo, não foram constatadas mudanças $(p>0,05)$ nas concentrações de sódio sanguíneo ${ }^{25}$.

A concentração de potássio não apresentou alterações após a prova de Ironman® 70.3 (Tabela 1), mantendo-se dentro dos níveis normais $(25-120 \mathrm{mmol} / \mathrm{L})$. Resultados semelhantes foram encontrados por Mara et a ${ }^{26}$, ao avaliarem 109 triatletas após a realização das provas de Ironman ${ }^{\mathrm{TM}}$. Esses mesmos autores afirmam que isso pode ter ocorrido devido à reposição das perdas deste eletrólito, provavelmente pela oferta disponibilizada ao longo da prova por meio de bebidas hidroeletrolíticas que costumam conter potássio suficiente para reposição deste mineral. Fato que pode ter ocorrido com a amostra do presente estudo, haja vista que durante todo o percurso da prova, todos os voluntários tiveram livre acesso ao consumo de água, repositores hidroeletrolíticos, bebidas glicosadas e comidas (ex: pão, banana, glicogel).

No presente estudo (Tabela 1), a concentração de cálcio permaneceu dentro dos valores normais de referência ( $\geq 45 \mathrm{mmol} / \mathrm{L})$, tanto antes quanto depois da prova de Ironman ${ }^{\circledR} 70.3$, sendo que houve um aumento de $15,1 \%(p<0,05)$ na concentração do cálcio após o término da prova. Esse aumento pode ser explicado pelo estresse gerado após o exercício físico prolongado, 0 qual provoca o aumento da liberação de cortisol que, consequentemente, diminui a absorção de cálcio no trato gastrointestina ${ }^{27}$.

No presente estudo, a CK total e suas frações (CK MM e CK MB) apresentaram valores dentro dos padrões de referência ${ }^{28}$. Os valores de referência da CK total em atletas variam entre 404 e $1479 \mathrm{U} / \mathrm{L}$, para CK MB são considerados valores normais até $25 \mathrm{U} / \mathrm{L}$ e a CK MM (95-100\% da CK total), levando em consideração homens e mulheres. Apesar dos triatletas amadores apresentarem valores do CK dentro dos padrões de referência, ao final da prova foi verificado um aumento $(p<0,05)$ em todas as análises quando comparadas aos valores pré prova. Esses achados corroboram com os estudos tendo como amostra triatletas ${ }^{29,30}$ e maratonistas ${ }^{31}$, que também apresentaram um aumento significativo da CK após a competição.

A CK total e a CK MM aumentaram 306,4 \% e 328,1\%, respectivamente, após 15 min do término da prova de triatlo. Considerando que a maior elevação da atividade de CK ocorre entre 24 e $72 \mathrm{~h}$ após a competição ${ }^{28,32}$ é possível que os atletas apresentem lesões músculo-esquelética mais acentuadas posteriormente. Entretanto, as alterações de CK MB (Tabela 1) indicam que não houve um desgaste do músculo cardíaco, pois sua concentração (11,8 U/L) ficou bem abaixo dos valores limítrofes de referência $(25 \mathrm{U} / \mathrm{L})^{28}$. Cabe ressaltar que a liberação de CK MM para a corrente circulatória é mais específica de sobrecarga muscular quando comparada a CK total e CK MB ${ }^{33}$.

A TBARs apresentou um aumento de 18,2\% ( $p<0,05$ ) após a prova (Tabela 1), indicando que houve uma produção elevada de espécies reativas de oxigênio. Resultados convergentes foram obtidos em 18 triatletas bem treinados, antes e após a prova do Ironman ${ }^{\mathrm{TM} 34}$, e em corredores após uma maratona na areia ${ }^{35}$ ao avaliarem corredores. Isto ratifica as evidências de que provas de longa duração provocam redução na capacidade antioxidante e danos oxidativos nos atletas, haja vista 0 fato da TBARs indicar uma elevada produção de espécies reativas de oxigênio, provocando danos musculares e alterações histológicas evidentes de dor muscular, dados não relatados, mas reportados por todos os sujeitos integrantes do estudo. 
Na análise da pressão arterial (Figura 1) foi verificada uma diminuição $(p<0,05)$ tanto na PAS quanto na PAD após a prova de Ironman® 70.3, ratificando os resultados obtidos em outros estudos feitos em triatletas de Ironman ${ }^{\top \mathrm{M} 25,36}$. Isso pode ser explicado pela vasodilatação após a realização de exercício extenuante e pelo aumento da concentração de lactato, o qual induz a diminuição da resistência vascular periférica refletindo-se no decréscimo da pressão arterial ${ }^{37}$. Sete atletas relataram calafrios, perda de força e/ou tonturas (dados não apresentados) ao final da prova, possivelmente ocasionada pela queda da pressão arterial, devido à redução do fluxo de sangue para os tecidos e, consequente, diminuição da oxigenação das células.

A presente investigação, do estado de hidratação, do dano muscular e dos indicadores de peroxidação lipídica, mostrou que os triatletas apesar de estarem hidratados e com as concentrações de eletrólitos dentro de níveis normais, apresentaram acentuado dano muscular (CK total e CK MM) e estresse oxidadivo. Assim, ressalta-se a importância da realização de análises bioquímicas periódicas durante todo o macrociclo e não apenas após a prova de Ironman® 70.3. Esse tipo de informação pode auxiliar na prevenção de lesões musculares e síndrome do sobretreinamento.

As principais limitações do presente estudo foram não ter padronizado o treino dos triatletas na semana que antecedeu a prova de Ironman® 70.3, não ter controlado a alimentação antes da prova, assim como o consumo de água, repositores hidroeletrolíticos, bebidas glicosadas e comidas (ex: pão, banana, glicogel) durante a realização da prova. Isso pode ter alterado as concentrações CK e TBARs, a resposta da hidratação e dos eletrólitos investigados no presente estudo. Além disso, a realização da análise da CK 24, 48 e 72h após a competição também poderia trazer informações relevantes a respeito destas variáveis, pois seu pico de concentração pode ocorrer dentro deste período ${ }^{28}$.

\section{Considerações Finais}

Com base nos resultados do presente estudo conclui-se que neste grupo de triatletas amadores a reposição hidroeletrolítica ao longo da prova foi suficiente para mantê-los hidratados.

Após a prova de Ironman ${ }^{\circledR} 70.3$ apresentaram desgaste da musculatura esquelética, formação de radicais livres e hipotensão, alterações esperadas para atletas desta modalidade esportiva.

Mesmo que estas alterações sejam esperadas, os participantes devem estar cientes quanto aos possíveis riscos à saúde e ser orientados quanto aos métodos de recuperação física e prevenção de lesões.

\section{Referências Bibliográficas}

1. Dallam GM, Jonas S, Miller TK. Medical considerations in triathlon competition: recommendations for triathlon organisers, competitors and coaches. Sports Med. 2005;35(2):143-61.

2. Almond CS, Shin AY, Fortescue EB, Mannix RC, Wypij D, Binstadt BA, et al. Hyponatremia among runners in the Boston Marathon. N Engl J Med. 2005 Apr 14;352(15):1550-6.

3. Goudie AM, Tunstall-Pedoe DS, Kerins M, Terris J. Exercise-associated hyponatraemia after a marathon: case series. J R Soc Med. 2006 Jul;99(7):363-67.

4. ADA/ACSM/DC. Joint Position Statement: nutrition and athletic performance. . Med Sci Sports Exerc. 2000 Dec;32(12):2130-45.

5. Long D, Blake M, McNaughton L, Angle B. Hematological and biochemical changes during a short triathlon competition in novice triathletes. Eur J Appl Physiol Occup Physiol. 1990;61(1-2):93-9.

6. Laursen PB, Suriano R, Quod MJ, Lee H, Abbiss CR, Nosaka K, et al. Core temperature and hydration status during an Ironman triathlon. Br J Sports Med. 2006;40(4):320-5.

7. Richter S, Betz C, Geiger H. Severe hyponatremia with pulmonary and cerebral edema in an Ironman triathlete. Dtsch Med Wochenschr. 2007;132(36):1829-32.

8. Silvestre JC, Juzwiak CR, Gollücke APB, Dourado VZ, D'Almeida V. Acute effect of a grape concentrate intake on oxidative stress markers in triathletes. Rev Bras Cineantropom Desempenho Hum. 2014;16(5):533-44.

9. Bloomer RJ, Goldfarb AH, McKenzie MJ. Oxidative stress response to aerobic exercise: comparison of antioxidant supplements. Med Sci Sports Exerc. 2006 Jun;38(6):1098-105.

10. Michailidis Y, Jamurtas AZ, Nikolaidis MG, Fatouros IG, Koutedakis Y, Papassotiriou I, et al. Sampling time is crucial for measurement of aerobic exercise-induced oxidative stress. Med Sci Sports Exerc. 2007 Jul;39(7):1107-113. 
11. Brancaccio P, Maffulli N, Limongelli FM. Creatine kinase monitoring in sport medicine. Br Med Bull. 2007;81-82:209-30.

12. Ministério da Saúde (BR). Conselho Nacional de Saúde. Diretrizes e normas regulamentadoras sobre pesquisa envolvendo seres humanos. Resolução 196/1996. Brasília: CNS; 1996.

13. Petroski EL, editor. Antropometria: Técnicas e padronizações. 3 ed. Blumenau: Nova Letra; 2007.

14. Draper HH, Hadley M. Malondialdehyde determination as index of lipid peroxidation. Methods Enzymol. 1990;186:421-31.

15. Rhea MR. Determining the magnitude of treatment effects in strength training research through the use of the effect size. J Strength Cond Res. 2004 Nov;18(4):918-20.

16. Barroso SS , Almeida RD , Gonzaga WS , Alves SR, Camerino SRAS, Lima RCP , Prado ES. Hydration status and cognitive-motor performance during a fast triathlon race in the heat. Rev Educ Fís/UEM, 2014;25(4):639-50.

17. Kao WF, Shyu CL, Yang XW, Hsu TF, Chen JJ, Kao WC, et al. Athletic performance and serial weight changes during 12- and 24-hour ultra-marathons. Clin J Sport Med. 2008;18(2):155-8.

18. Pastene J, Germain M, Allevard AM, Gharib C, Lacour JR. Water balance during and after marathon running. Eur J Appl Physiol Occup Physiol. 1996;73(1-2):49-55.

19. Knechtle B, Knechtle P, Rosemann T, Oliver S. A Triple Iron triathlon leads to a decrease in total body mass but not to dehydration. Res Q Exerc Sport. 2010;81(3):319-27.

20. Knechtle B, Baumann B, Wirth A, Knechtle P, Rosemann T. Male ironman triathletes lose skeletal muscle mass. Asia Pac J Clin Nutr. 2010;19(1):91-7.

21. Speedy DB, Noakes TD, Kimber NE, Rogers IR, Thompson JM, Boswell DR, et al. Fluid balance during and after an ironman triathlon. Clin J Sport Med. 2001;11(1):44-50.

22. Herbst L, Knechtle B, Lopez CL, Andonie JL, Fraire OS, Kohler G, et al. Pacing strategy and change in body composition during a deca iron triathlon. Chin J Physiol. 2011;54(4):255-63.

23. Howanitz JH, Howanitz PJ. Evaluation of serum and whole blood sodium critical values. Am J Clin Pathol. 2007 Jan;127(1):56-9. 24. Bürger-Mendonça M, Bielavsky M, Retondaro-Barbosa FC. Significant sodium plasma reduction after half-ironman triathlon in brazilian triathletes. Journal of Human Sport and Exercise online. 2009;4(3):246-53.

25. Sharwood KA, Collins M, Goedecke JH, Wilson G, Noakes TD. Weight changes, medical complications, and performance during an ironman triathlon. Br J Sports Med. 2004 Dec;38(6):718-24.

26. Mara LS, Lemos R, Brochi L, Rohlfs ICPM, Carvalho T. Acute hydroelectrolytic changes occurred in the Brazilian Ironman Triathlon. Brazilian journal of sports medicine. 2009;13(6):397-401.

27. Sattler AM, Schoppet M, Schaefer JR, Hofbauer LC. Novel aspects on RANK ligand and osteoprotegerin in osteoporosis and vascular disease. Calcif Tissue Int. 2004 Jan;74(1):103-6.

28. Wyss M, Kaddurah-Daouk R. Creatine and creatinine metabolism. Physiol Rev. 2000 Jul;80(3):1107-213.

29. Shave R, Dawson E, Whyte G, George K, Gaze D, Collinson P. Altered cardiac function and minimal cardiac damage during prolonged exercise. Med Sci Sports Exerc. 2004 Jul;36(7):1098-103.

30. Machado CN, Gevaerd MS, Goldfeder RT, Carvalho T. Exercise effects on serum levels of creatine kinase in ultradistance triathletes in the course of a competition period. Br J Sports Med. 2010;16(5):378-81.

31. Siqueira LO, Muccini T, Agnol ID, Filla L, Tibbola P, Luvison A, et al. Biochemist plasmatic and urinary parameters analisys in marathon athletes. Arq Bras Endocrinol Metab. 2009;53(7):844-52.

32. Lehmann M, Gastmann U, Petersen KG, Bachl N, Seidel A, Khalaf AN, et al. Training-overtraining: performance, and hormone levels, after a defined increase in training volume versus intensity in experienced middle- and long-distance runners. Br J Sports Med. 1992 Dec;26(4):233-42.

33. Echegaray M, Rivera MA. Role of creatine kinase isoenzymes on muscular and cardiorespiratory endurance: genetic and molecular evidence. Sports Med. 2001;31(13):919-34.

34. Pinho RA, Silva LA, Pinho CA, Scheffer DL, Souza CT, Benetti M, et al. Oxidative stress and inflammatory parameters after an Ironman race. Clin J Sport Med. 2010 Jul;20(4):306-11.

35. Machefer G, Groussard C, Rannou-Bekono F, Zouhal H, Faure H, Vincent S, et al. Extreme running competition decreases blood antioxidant defense capacity. J Am Coll Nutr. 2004 Aug;23(4):358-64.

36. Gratze G, Rudnicki R, Urban W, Mayer H, Schlogl A, Skrabal F. Hemodynamic and autonomic changes induced by Ironman: prediction of competition time by blood pressure variability. J Appl Physiol. 2005 Nov;99(5):1728-35.

37. Clifford PS, Hellsten Y. Vasodilatory mechanisms in contracting skeletal muscle. J Appl Physiol. 2004 Jul;97(1):393-403. 


\section{Kenji Fuke}

Endereço para correspondência - Rua Maria Tereza Mallmann, nº 77, Bloco CJ 1 - ap 202 , Moinhos, CEP: 95900-000, Lajeado, RS, Brasil.

E-mail: knjfuke@yahoo.com.br

Lattes: http://lattes.cnpq.br/9259462602475346

Vanderson Luis Moro - vandersonmoro@hotmail.com

Camila de Campos Velho Gewehr - camigewehr@hotmail.com

Maria Fátima Glaner - mfglaner@gmail.com

Enviado em 19 de maio de 2014.

Aceito em 13 de agosto de 2015. 